\title{
Conditions sociales, registres de vulnérabilité et territoires de vie dans la France d'aujourd'hui
}

Social conditions, registers of vulnerability and living areas in todays's France

Hervé Marchal

\section{OpenEdition}

1 Journals

Édition électronique

URL : https://journals.openedition.org/popvuln/1101

DOI : $10.4000 /$ popvuln. 1101

ISSN : 2650-7684

Éditeur

LIR3S - Laboratoire Interdisciplinaire de Recherche "Sociétés Sensibilités Soin" (UMR 7366 CNRS-uB)

Édition imprimée

Date de publication : 1 mars 2019

Pagination : 19-39

ISBN : 978-2-918173-26-7

ISSN : 2269-0182

Référence électronique

Hervé Marchal, « Conditions sociales, registres de vulnérabilité et territoires de vie dans la France d'aujourd'hui », Populations vulnérables [En ligne], 5 | 2019, mis en ligne le 26 mai 2020, consulté le 26 février 2022. URL : http://journals.openedition.org/popvuln/1101 ; DOI : https://doi.org/10.4000/ popvuln. 1101

Ce document a été généré automatiquement le 26 février 2022.

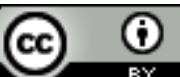

Les contenus de la revue Populations vulnérables sont mis à disposition selon les termes de la Licence Creative Commons Attribution 4.0 International 


\section{Conditions sociales, registres de vulnérabilité et territoires de vie dans la France d'aujourd'hui}

Social conditions, registers of vulnerability and living areas in todays's France

Hervé Marchal

\section{Introduction}

1 Diffusée dans la littérature psychiatrique et psychologique à partir des années 1970 en lien avec les notions de fragilité et de dépendance, puis dans des disciplines aussi diverses que le management des catastrophes, l'économie du développement, les sciences environnementales, de la santé et de la nutrition ou encore la statistique (Brodiez-Dolino, 2016), la notion de vulnérabilité s'est aujourd'hui imposée dans de nombreuses disciplines. C'est notamment le cas en philosophie durant ces dernières années (Tronto, 2009; Butler, 2010; Le Blanc, 2011). D’une façon générale, comme le remarque Marc-Henri Soulet :

tout le monde s'est approprié les vertus de la vulnérabilité. La vulnérabilité autorise toutes les relectures de notre dynamique sociétale, chacun y recourant tantôt pour asseoir de nouveaux mots d'ordre préventifs, tantôt pour fonder une orientation normative positive pour autrui, tantôt pour ré-agencer les logiques d'action en direction des plus fragiles, tantôt pour ancrer une conception renouvelée de notre vie commune [...], elle est devenue une catégorie dominante d'expression des difficultés à être en société autant qu'une catégorie agissante (Soulet, 2014, p. 59)

D'aucuns diront que ce que le concept de vulnérabilité a gagné en surface, il l'a perdu en précision. Mais au-delà du fait de s'inscrire dans une longue tradition de compréhension du vécu des plus précaires et des plus démunis, la notion de vulnérabilité comporte plusieurs volets à la portée heuristique non négligeable. D'abord, elle autorise à prendre en compte, en la matière, à la fois les facteurs positionnels et individuels, ou sociaux et personnels ${ }^{1}$ si l'on préfère, en invitant «à mieux comprendre la dialectique entre grandes positions structurelles et états sociaux 
effectifs des acteurs, c'est-à-dire les stratégies qu'ils mettent - ou non - en place pour se prémunir contre les risques, les accidents, les déclassements, en bref, contre tout ce qu'ils ressentent comme source possible de vulnérabilité " (Martuccelli, 2014, p. 38). Ensuite, la vulnérabilité peut renvoyer non seulement à des dimensions sociales (relationnelles, économiques, culturelles, symboliques, etc.), mais également à des dimensions internes aux individus d'ordre bio-psychologique et/ou à des conditions externes d'ordre climatique (tsunamis, séismes, etc.), morphologique (absence de services publics dans un territoire excentré, faible accessibilité routière, ferroviaire ou aérienne, etc.), géopolitique (guerres, génocides, etc.). Cette acception large raisonne avec les travaux des philosophes soucieux de rappeler à quel point l'être humain est, du fait même de sa condition, vulnérable physiquement, psychiquement et socialement. Puis, parallèlement à cette conception large et plurielle des sources possibles de vulnérabilité, la notion recouvre l'intérêt, dès lors qu'une certaine prudence épistémologique est de mise, de renvoyer davantage à des situations, des moments ou à des territoires qu'à des personnes en tant que telles toujours susceptibles d'être réduites à leur seul état de vulnérabilité; ce qui très souvent se traduit par une réification de ces mêmes personnes alors qualifiées radicalement de "vulnérables » comme si elles n'étaient que cela. Enfin, parallèlement à cette conception situationnelle et spatialisée de la vulnérabilité, le terme invite, dans une visée dynamique, à analyser les "parcours de vie» (Elder, 1994) marqués par des processus de négociation, des tournants biographiques, des contraintes surmontables et insurmontables, des épreuves qui construisent l'individu tout autant qu'elles le déconstruisent - sur le plan identitaire notamment-, ou encore à des sorties de situations de vulnérabilité rappelant, au demeurant, combien la vulnérabilité ne conduit pas ipso facto à l'exclusion.

3 Dans ce sens, nous adopterons la définition selon laquelle :

la vulnérabilité renvoie de manière générale à la situation d'individus ou collectifs qui, du fait de certaines caractéristiques propres et de circonstances ou de facteurs extérieurs, sont exposés à une altération significative de leurs conditions d'existence, de leurs capacités de prendre en charge leurs besoins et de faire face aux aléas de la vie, de leurs possibilités d'action et d'évolutions futures (Bickel, 2015, p. 300)

4 Fort de cette définition, nous développerons dans le cadre de cet article des liens analytiques entre des formes de vulnérabilités et des territoires précis. Nous nous appuierons sur des recherches empiriques que nous avons réalisées au cours de ces six dernières années. Les terrains de recherche retenus se situent en France et portent aussi bien sur des zones résidentielles du périurbain éloigné d'une métropole régionale, sur une tour déshéritée située dans une cité d'habitat social ou sur un bidonville ${ }^{2}$. Ils peuvent être, au regard de leurs caractéristiques, considérés comme des révélateurs des formes plurielles que prend la vulnérabilité dans la société française actuelle, sans bien évidemment prétendre à une quelconque exhaustivité. À cet égard, dans le cadre de cet article qui se veut synthétique puisque s'appuyant sur plusieurs recherches, nous ne mettrons en évidence que des formes manifestes de vulnérabilité, celles-là même qui ont "parlé » le plus à travers les entretiens et les observations réalisés. Cela étant précisé, conformément à nos prémisses théoriques, il s'agira d'être sensible à la pluralité des sources et des formes de vulnérabilité. Aussi identifierons-nous, de façon inductive, différents registres de vulnérabilité. 
5 Parce que ces différents registres en disent long sur l'existence de fortes disparités non seulement spatiales mais aussi sociales, nous donnerons de la hauteur à notre propos en inscrivant les formes de vulnérabilités identifiées empiriquement dans des conditions sociales idéal-typiques: l'intro-socialité, l'hétéro-socialité et l'extrosocialitée ${ }^{3}$. Ces dernières révèlent combien la "société » française contemporaine ne peut être assimilée à une seule condition sociale mais bien à plusieurs, témoignant de profondes divergences socio-spatiales (Marchal, 2017). L'option d'une telle approche idéal-typique vise à opérer une montée en généralité théorique pour y voir plus clair dans un monde dont la complexité ne semble pouvoir être saisie qu'en l'identifiant à partir de traits saillants. Cette façon de procéder s'avère d'autant plus pertinente quand elle ne propose pas de caractéristiques trop abstraites détachées de toute réalité vécue. Souhaitant poser des abstractions concrètes, le conçu analytique se veut ici, en dépit de sa volonté généralisante, en lien très étroit avec le vécu social, et même avec le perçu singulier - le sentir - de tout un chacun.

6 Nous commencerons par développer la condition intro-sociale pour montrer comment les zones périurbaines excentrées, typiques de l'intro-socialité, sont notamment le théâtre de vulnérabilités d'ordre morphologique, relationnel et narratif. Puis, nous préciserons les traits saillants de la condition hétéro-sociale pour souligner combien celle-ci peut se traduire par des vulnérabilités identitaires, relationnelles, existentielles et fictionnelles, comme nous l'avons observé dans une tour déshéritée d'une cité d'habitat social. Enfin, nous analyserons la condition extro-sociale à partir d'une recherche réalisée au sein d'un bidonville afin de montrer à quel point dominent ici des vulnérabilités relevant des registres du matériel, du corporel, du culturel et du représentationnel.

\section{La condition intro-sociale}

7 Si une nouvelle élite urbaine internationale se dessine, élite composée pour l'essentiel de hauts cadres pleinement intégrés dans les échanges et les flux mondiaux, il reste qu'en dehors de cette « classe internationale » et circulante (Wagner, 2007) la plupart des individus continuent de vivre plus ou moins à l'écart des réseaux planétaires. Il en est ainsi des classes moyennes au sens large (Damon, 2013). Ce qui est dès lors au centre de l'analyse, c'est l'ordinarité de la vie urbaine. En effet, les ressortissants de la condition intro-sociale correspondent à ces individus engagés dans des mobilités quotidiennes à l'échelle d'une agglomération ou d'une aire urbaine, mobilités nécessaires pour conjuguer vie familiale, vie professionnelle, éducation des enfants, loisirs, etc.

8 Dans la condition intro-sociale, on se frotte à autrui à travers de multiples espaces fréquentés jour après jour, d'où l'engagement dans une socialisation par frottements. Aussi l'épreuve sociale typique renvoie, en l'occurrence, au fait d'assumer une quotidienneté répétitive à travers des mobilités récurrentes. Comme dans l'hyperurbanité, il est question ici d'un capital de mobilité, mais localisé et non globalisé : on se meut à l'échelle de l'agglomération, de l'aire urbaine, et non à l'échelle de la planète, loin de là. L'horizon de sens ne renvoie pas au monde, mais bien plus aux territoires du quotidien. Ici, plus que l'avion, c'est bien souvent le train et peut-être plus encore l'automobile qui règnent en maître dans les parcours quotidiens de ceux s'apparentant souvent à des « navetteurs » (Le Breton, 2008). 


\section{Des vulnérabilités d'ordre morphologique et relationnel}

9 À cet égard, les espaces résidentiels privilégiés et caractéristiques des « intro-sociaux » ${ }^{4}$ correspondent aux zones pavillonnaires du périurbain proche et éloigné, même si les individus concernés peuvent habiter en centre-ville, en banlieue ou encore au sein du rural plus ou moins urbanisé. Cela étant précisé, c'est certainement au sein des couronnes périurbaines les plus éloignées des centres-villes que se donnent le plus à voir des formes de vulnérabilités intro-sociales, comme nous avons pu l'observer dans le périurbain éloigné de la ville de Nancy (située au nord-est de la France) à partir de recherches de terrain menées entre 2012 et 2016. Ces recherches sont centrées sur les discours de justification recueillis a posteriori quant aux choix résidentiels de familles modestes et récemment installées dans un pavillon ou dans une maison à rénover.

10 Les espaces périurbains observés se caractérisent par l'installation d'habitants modestes. À ce propos, les territoires que nous avons investis se distinguent des couronnes périurbaines classiques davantage proches de la ville centre, et ce d'autant plus qu'ils n'accueillent pas de vastes lotissements pavillonnaires, ni d'importantes zones commerciales, pas plus que de nombreux espaces dédiés à la petite et moyenne industrie, à la logistique et aux pépinières d'entreprises innovantes. Lorsque l'on pénètre dans les villages éloignés de la centralité nancéienne, ce qui s'impose au regard, c'est d'abord la présence de nombreuses automobiles qui occupent trottoirs et abords des rues. C'est également des vitrines aux rideaux de fer tirés depuis longtemps, des pavillons récents et modestes construits à l'écart du bourg initial, autant de signes d'une vie villageoise en profonde mutation.

11 Après avoir décrit longuement leur projet de résider dans un pavillon loin de la ville et après avoir vanté les avantages de la campagne à travers une narration qui gomme les doutes et les incertitudes, de nombreux informateurs vivant en lotissement se montreront au fur et à mesure de l'entretien plus ambivalents et finiront par dénoncer les carences de la vie excentrée de toute centralité urbaine. En effet, ces derniers n'hésiteront pas à souligner combien leur pavillon, situé dans un lotissement avec « des maisons toutes pareilles », "sans âme » et "sans originalité ", se trouve éloigné, voire coupé (par une route, une voie ferrée, un bois ou encore un pont) du centre du village. Celui-ci est alors vu comme un véritable espace de sociabilité si bien que la vie sociale y est imaginée plus intense et amicale, quand elle n'est pas fantasmée. Dans ces conditions, nos informateurs se rendent compte qu'ils ne sont pas partie prenante de la vie quotidienne du village, des petites rencontres informelles, des conversations rituelles et autres scènes de sociabilité concrètes, ici au coin d'une rue, là devant la boulangerie, ailleurs sur le perron de la mairie. Autant dire que nombre d'habitants que nous avons rencontrés ont conscience de l'isolement social dans lequel ils se trouvent bien qu'ayant élu domicile dans un village.

12 Ce sentiment d'être isolé est renforcé par la nécessité de recourir quotidiennement à la voiture étant donné l'éloignement des commerces par rapport à la maison. En effet, à la différence de la marche ou de la bicyclette, l'automobile ne permet pas de relations sensibles avec les autres habitants, tout au plus autorise-t-elle un simple signe de la main éphémère, comme le rappelle cette habitante : «Dans la rue, les gens ne font que passer en voiture, ils roulent bien trop vite pour qu'on ait le temps de se dire bonjour.

Populations vulnérables, 5 | 2019 
On est chacun dans notre voiture... il ne se passe rien, on ne se parle pas. » (mariée, mère de 4 enfants, vendeuse, résidant au sein d'un pavillon depuis 6 ans).

Nos informateurs ont tenu à souligner que les coupures spatiales s'accompagnent de clivages sociaux revêtant une expression toute particulière au moment des échéances électorales et, partant, de la composition des listes en vue des élections municipales. À entendre les habitants rencontrés, de telles tensions vont de pair avec des oppositions entre les "gens du lotissement " et les "vieux du village", autrement dit entre les « anciens » et les « nouveaux ». Cela n'est pas sans rappeler les logiques de démarcation et les pratiques d'ostracisme bien identifiées dans les travaux désormais classiques de Norbert Elias et John L. Scotson (1997). Au regard de cette solidarité villageoise qui n'est pas au rendez-vous, comment s'étonner que nous ayons souvent entendu en fin d'entretien des propos laissant transparaître de nombreuses frustrations et un fort désenchantement rappelant qu'ici la vulnérabilité, certes moins tragique que dans d'autres cas, prend son origine dans la morphologie des villages observés, entendons dans leurs caractéristiques spatiales renvoyant à des frontières non seulement « objectives» (économiques, culturelles, etc.), mais également symboliques, morales et donc relationnelles (Lamont, Molnär, 2002).

\section{Des vulnérabilités d’ordre narratif}

Si les entretiens ont été l'occasion de mettre en avant, pour les familles qui ont "choisi » de rénover une maison en cœur de village faute bien souvent de pouvoir acquérir un pavillon, la fierté de "faire soi-même les travaux ", l'importance de la proximité avec la nature et l'authenticité des relations sociales, il reste qu'au-delà des récits donnant à voir une quotidienneté idéale, les habitants ont souvent réajusté, là encore, leurs propos au fur et à mesure de l'avancée des entretiens.

Force est d'observer, par exemple, que beaucoup supportent mal, avec les années qui passent, de voir leur maison toujours en chantier. Combien de fois n'avons-nous pas entendu à ce sujet des épouses, visiblement lasses, se plaindre des travaux qui n'en finissent pas ou encore de la poussière qui s'infiltre partout : "Vous savez, une vieille maison, c'est vrai que ça peut être beau, mais pour ça il faut que les travaux soient finis. Chez nous ce n'est pas le cas, moi j'en ai ras le bol, et si c'était à refaire, j'y réfléchirais à deux fois!» (femme, pacsée, 2 enfants, au chômage depuis 1 an, vit dans son village depuis 7 ans). Ce sentiment que « rien n'avance » est loin d'être anodin étant donné que bien souvent l'argent et le temps manquent cruellement pour réaliser son projet de rénovation. Or, le temps qui manque au quotidien parce qu'il faut emmener les enfants au club sportif ou à la répétition de musique, les week-ends trop courts pour faire les courses de la semaine, et les économies impossibles à réaliser en raison du coût d'entretien élevé des voitures, compromettent sérieusement l'avancée des travaux. D'où l'apparition progressive d'une lassitude et d'un découragement consécutifs au fait de ne pas être en mesure d'aménager à sa guise la maison dans laquelle on s'est pourtant projeté. Les entretiens ont révélé chez certains habitants une fatigue de ne pas se sentir complètement chez soi, un essoufflement comme pour cet homme qui admettra au bout d'une heure d'entretien: "Vous savez, vivre toujours dans les travaux, c'est pas drôle du tout et pour ma femme c'est même exaspérant. On a, vous savez, le sentiment qu'on n'y arrivera pas avec les enfants à s'occuper et avec les trajets à faire tous les jours. Peut-être qu'on a vu trop grand, qu'on n'a pas assez réfléchi car 
on est loin de tout...» (homme, marié, 2 enfants, cadre dans un institut médicopédagogique, vit dans son village depuis 6 ans).

De fait, il faut compter avec les différends susceptibles d'apparaître au sein des familles, que cela soit au sein du couple ou entre les enfants et les parents : «Les travaux pas finis, comme ça gonfle tout le monde, ça devient des sujets d'engueulade. Mon mari n'arrête pas de me dire qu'il n'a pas le temps, qu'il a autre chose à faire sur son ordinateur et qu'il aimerait bien regarder ses matchs à la télé. Et les enfants, ils me bassinent que la chambre n'est pas belle, et quand ils font venir des petits copains, ils aiment pas trop...» (femme, mariée, 3 enfants, assistante maternelle, vit dans son village depuis 9 ans). Ces propos se comprennent d'autant plus que la finition de la maison, sa décoration intérieure et son aménagement extérieur se donnent à voir aux autres, notamment aux voisins et aux amis qui ne peuvent qu'inférer, au regard du "chantier permanent", un manque flagrant de moyens financiers. C'est bien in fine cela qui affecte nos informateurs étant donné qu'il s'agit derrière tout cet inachevé de laisser parler les choses de manière peu valorisante : une porte d'entrée hors d'usage, une chambre sans papier peint, une cuisine sans équipements électroménagers intégrés sont en effet autant de " porte-parole » qui parlent de trop (Callon, 1986).

$\mathrm{Au}$ regard des propos de nos informateurs, force est de constater que les discours recèlent ici, au-delà des rhétoriques de "façade», des sentiments empreints de déception, de dépit et de regret. Les habitants récemment implantés aux confins de l'agglomération de Nancy que nous avons interviewés se rendent compte, au fur et mesure du temps, que leur choix résidentiel s'avère moins idéal qu'il n'en avait l'air de prime abord. Aussi admettent-ils, avec pudeur et non sans une certaine amertume, qu'ils n'ont en réalité ni les avantages de la ville, puisqu'ils sont loin des structures médicales, des bassins d'emploi, des équipements scolaires et culturels, des centres commerciaux, etc., ni les avantages de la campagne, étant donné qu'ils ne sont pas intégrés dans un réseau de sociabilité local. Se dessinent ici les contours d'une vulnérabilité narrative renvoyant à une difficulté à raconter son choix résidentiel sous des auspices favorables et à construire un récit supportable de son quotidien et de son avenir.

\section{La condition hétéro-sociale}

Parallèlement, il faut compter avec l'hétéro-socialité qui concerne notamment les habitants de certaines cités d'habitat social en difficultés ou les résidents d'un habitat privé déshérité - habitat social de fait - pouvant se trouver aussi bien en ville centre, en banlieue qu'au sein de zones semi-urbaines ou rurales.

Les « hétéro-sociaux " s'apparentent souvent à la figure de l'individu «insulaire » (Le Breton, 2006) dont la caractéristique essentielle est de ne pas se représenter le territoire comme un ensemble continu et solidaire, mais au contraire de voir l'espace organisé autour de zones peu accessibles, voire pas du tout. Ils sont engagés dans une socialisation par évitement subie dans la mesure où ils ne choisissent pas leur place : de vivre là où il est pourtant peu légitime de vivre. Aussi les épreuves auxquelles ils doivent faire face renvoient-elles à la relégation, à la disqualification ou encore au racisme (Paugam, 1997 ; Lapeyronnie, 2008). C'est dès lors un " capital d'autochtonie » (Renahy, 2005) par défaut qui peut dominer ici étant donné que l'attachement à son lieu de vie, aussi stigmatisé soit-il, permet de revendiquer un attachement, un ancrage 
socio-spatial, voire une appartenance dans le meilleur des cas. Parfois, pour les plus jeunes des quartiers d'habitat social déshérités notamment, c'est d'un «capital guerrier » dont il s'agit tant le recours à des tactiques de détournement, à l'entretien d'un réseau de relations locales et à la force physique s'impose (Sauvadet, 2006).

\section{Des vulnérabilités d'ordre identitaire et relationnel}

Pour beaucoup, la condition hétéro-sociale interdit l'expérience d'un rapport positif à soi et se traduit par de la honte. Il en résulte un sentiment d'être en dehors de la société qui s'accompagne d'une fatigue révélatrice d'un quotidien lourd à supporter. Apparaît alors une tendance à se replier sur soi et son logement. À la fatigue s'ajoute ainsi une invisibilité qui prive l'individu de lui-même, car ne plus être présent dans le monde revient à déléguer aux autres la construction de sa propre image (à la presse notamment...).

Cette vulnérabilité d'ordre identitaire, nous l'avons observée sous une forme radicale lors d'une recherche réalisée en 2011 dans une tour d'habitat social très paupérisée. La tour en question est implantée au sein d'un quartier d'habitat social théâtre depuis plusieurs années d'une importante entreprise de rénovation urbaine financée par l'Agence nationale de rénovation urbaine (ANRU). Connaissant une concentration de locataires sans emploi - au moment de l'enquête ils n'étaient que 12 sur 52 à occuper un emploi -, la tour n'est pas très visible dans le quartier puisqu'excentrée. Aussi le hall d'entrée était-il constamment envahi, au moment de la recherche, par une « ambiance » olfactive mêlant odeurs de tabac, d'alcool, de cannabis et d'urine.

Il ressort de nos observations que les habitants du quartier qui ne vivent pas dans cette tour-reliquat, au mieux y passent en voiture, au pire ne s'y rendent jamais, si bien qu'ils ne connaissent que très mal l'endroit. Cette tour joue de fait un rôle d'utilité identitaire pour les habitants du quartier qui n'y habitent pas, étant donné qu'elle permet à ces derniers de se sentir moins paumés, moins "pauvres ", moins " cas-soc'" que ceux qui habitent là : dans «la tour des soulards, des camés » comme le dira un locataire de la tour en conflit ouvert avec nombre de ses voisins et visiblement excédé. Aussi cette tour permet-elle à beaucoup d'autres résidents du quartier, à travers un jeu de miroir, de se construire une image de soi positive en trouvant de quoi se comparer à plus mal loti que soi.

Lorsqu'un " hétéro-social » évolue au quotidien dans un territoire proposant si peu de références positives et contrariant de fait la formulation de projets de sens, il est contraint de mobiliser des supports identitaires relatifs à son passé susceptibles d'être en inadéquation avec ce que demandent les situations vécues, d'où le sentiment « d'être à côté de la plaque » ou " de ne pas être à la hauteur ». De ce point de vue, le rythme et l'intensité de la vie urbaine risquent fort d'amplifier un effet d'hystérésis identitaire vecteur d'une certaine inertie de soi et $d u$ soi, continuant à perpétuer des supports de sens inadéquats par rapport aux situations d'aujourd'hui, ce qui est susceptible de rendre la vie insupportable stricto sensu. 


\section{Des vulnérabilités d'ordre existentiel et fictionnel} telle situation sociale-limite, par une déshumanisation de l'autre : de celui qui incarne en négatif sa propre situation, qui rappelle chaque jour que l'on n'a pas été en mesure d'échapper à son existence, d'offrir à ses enfants de meilleures conditions de vie. C'est dire si l'utilité identitaire de l'autre n'est ici réelle que s'il est déshumanisé, ni plus, ni moins. On atteint en quelque sorte le degré ultime et tragique de la démarcation sociale. Tragique dans le sens où une telle situation révèle combien la composition de son intégrité personnelle demeure vulnérable au regard d'un autre jugé d'autant plus décadent qu'il rappelle, d'une part, sa propre condition sociale et, d'autre part, son impossibilité à être totalement indifférent à lui, tant l'ignorer " est encore une manière de réagir à sa présence " (Flahault, 2002, p. 374). Dans ces conditions, il n'y a rien d'étonnant à ce que les contours de son propre avenir s'effacent. L'absence de fictions identitaires (Markus, Nurius, 1986) traduit une impossibilité à se réaliser, à réaliser ses propres scénarios existentiels. Ici, chacun est à tout moment vulnérable quant à une impossibilité à rêver, à se projeter, à donner un sens à sa vie. Vulnérabilités 
existentielles et fictionnelles se confondent alors au point de se renforcer mutuellement.

\section{La condition extro-sociale}

Plus encore que la condition de l'hétéro-socialité, c'est sûrement celle de l'extrourbanité qui incarne le plus les logiques d'exclusion et de chosification de l'être humain. Les «extro-sociaux » sont typiquement incarnés par les Gens du voyage, les Roms, les immigrés clandestins, les Sans domicile fixe, etc.

Aussi étonnant que cela puisse paraître, la condition de l'extro-socialité entretient des affinités avec celle de l'hyper-urbanité, notamment en ce qui concerne un régime de forte mobilité (Tarrius, 2002). Mais les extro-sociaux se voient limités dans leurs déplacements pour des raisons économiques - pouvoir d'achat - et surtout politiques passeports, visas, frontières -, notamment depuis les attentats du 11 septembre 2001 suivis d'un durcissement des politiques sécuritaires et d'un renforcement des barrières migratoires, quand ce n'est pas leur militarisation (postes frontière armés, brigades mobiles, grillages électrifiés, etc.). Ce qui caractérise les extro-sociaux, c'est une socialisation par errements allant de pair avec les épreuves de la clandestinité, de la non-existence, de l'inexistence juridique, de la débrouille, du déracinement, de la traçabilité et du filtrage. C'est dire si les extro-sociaux sont les plus concernés et éprouvés par l'érection de murs physiques, juridiques et politiques: en un mot par l'« infamie " désignant des existences racontées exclusivement à travers les traces qu'elles ont laissées lors de leur confrontation avec le pouvoir (Foucault, 1988).

Les horizons de sens sont logiquement marqués par une logique de vie faite de déracinement et de subsistance. Quant au rapport à soi, il se caractérise typiquement par la visée d'une autre existence, par des fictions identitaires mettant en scène un ailleurs sécurisant ${ }^{5}$; ce qui se comprend au regard des zones d'habitat des extrosociaux: camps de réfugiés, campements divers, habitats de fortune, bidonvilles, squats, etc., autant de "hors-lieux» faits d'incertitudes, de précarité et d'exclusion (Agier, 2015).

\section{Des vulnérabilités d'ordre corporel et matériel}

Nous avons été en mesure d'observer de près cette condition extro-sociale dans un bidonville situé dans la banlieue proche de la ville de Nancy. Entre 2013 et 2016, nous nous sommes en effet immergés ${ }^{6}$, suivant une démarche ethnographique, dans une zone d'habitat bidonvillisée comptant quelques 50 personnes d'origine roumaine. Au sein du bidonville, réussir à mettre en place un espace de salubrité relève d'un combat quotidien perdu d'avance... Femmes, hommes et enfants participent tous d'une façon ou d'une autre au maintien de la propreté. La circulation constante entre les caravanes et les cabanes charrie de la terre, de la boue et autres papiers ou feuilles mortes. Il est donc nécessaire de passer sans cesse le balai pour endiguer l'arrivée constante d'éléments impropres. Au sol, à la sortie des maisons et sous les auvents, de gros tapis de linoléum facilitent le nettoyage. À l'intérieur des cabanes, pour apporter un peu de confort, des tapis en tissu ont été superposés à même le sol. Ils ajoutent un peu de chaleur en renforçant une sensation de " cocon ». Mais leur nettoyage est un fardeau supplémentaire... Les murs des caravanes ou des cabanes en bois sont recouverts d'un 
papier-peint remplacé tous les six mois à cause de la prolifération de moisissures rappelant l'humidité rémanente. Étant donné le nettoyage quotidien que nécessite la vie sur le camp, les habitants n'hésitent pas à jeter directement par terre papiers plastiques et autres épluchures de fruit. Le sol incarne alors de façon paroxystique une surface impropre : tout ce qui le touche devient souillé.

31 L'absence d'eau arrivant directement sur le bidonville accentue les difficultés. En fait, à bien y regarder, une danse des caddys chargés de bidons d'eau de 25 litres anime constamment les journées. Deux bornes à incendie se trouvent à 300 mètres de chaque côté du bidonville. Comme on s'en doute, le travail est pénible du fait de son aspect répétitif et éreintant en raison du poids des bidons. Pour les lessives, une grande casserole d'eau est constamment chauffée par un feu de bois situé au milieu de l'enceinte. Elle fait partie de la vie collective : chacun l'alimente en combustibles pour garder l'eau chaude durant toute la journée. Dans de petites piscines en pastique pour enfant - piscines en forme de coquillage -, on fait tremper des vêtements avant de les frotter à la main. Les problèmes aux poignets dus à cette tâche sont courants chez les femmes. Ici plus qu'ailleurs, la vulnérabilité des vies est avant tout d'ordre matériel mais aussi et surtout corporel tant la chair est marquée par le froid, l'humidité, les maladies, les plaies infectées, la fatigue, la malnutrition, etc. La saleté, la poussière ou encore la boue omniprésente collent aux habitants, marquent les corps. La boue sur les chaussures est à n'en pas douter l'une des expressions les plus fortes de la souillure dont il faut se débarrasser dès lors que l'on sort du bidonville (Sayad, 1989).

\section{$X$. Des vulnérabilités d'ordre culturel et représentationnel}

32 Parallèlement, la vulnérabilité a trait aux représentations négatives dont l'un des ressorts réside dans l'affirmation d'une culture peu assimilable dont les habitants des bidonvilles seraient les dépositaires. Le 24 septembre 2013, Manuel Valls, comme tant d'autres acteurs politiques, allait dans ce sens en expliquant sur les ondes de France Inter que « ces populations ont des modes de vie extrêmement différents des nôtres et qui sont évidemment en confrontation ». Dès lors, la culture rom est opposée frontalement à celle du pays d'accueil. C'est là tout le paradoxe du relativisme culturel ambiant dans notre société : de l'idée que les cultures ne peuvent se juger et s'évaluer l'une l'autre, on passe très facilement à l'idée qu'elles ne peuvent se mélanger, d'où l'idée très répandue d'un « choc des cultures ".

Cette manière de considérer les habitants des bidonvilles procède en fait d'un renversement de la question. Car les conditions de vie des Roms ne sont alors pas expliquées par les discriminations subies et des facteurs structurels (ostracismes en tous genres, inégalités, exclusion, etc.), mais par une sorte de prédisposition culturelle à vivre à l'écart des autres populations et même à habiter en bidonville. Cette croyance culturaliste produit ses propres effets dans la mesure où elle va de pair avec une réelle marginalisation sociale des populations identifiées comme Roms qui, en retour, tendent à se replier sur une culture alors revendiquée, telle une prophétie auto-réalisatrice. La vulnérabilité est en l'occurrence d'ordre représentationnel dans la mesure où les habitants finissent plus ou moins, et selon les situations, par s'identifier à l'identité culturelle assignée par autrui et censée leur coller à la peau. C'est notamment le cas lorsque les travailleurs sociaux entendent faire reconnaître des particularismes 
culturels « roms » d'habitants pourtant nés en France. Il suffit de penser ici aux enfants qui, lors de fêtes scolaires ou d'événements médiatisés, sont invités à s'exprimer sur leur culture rom à laquelle ils s'identifient alors qu'ils n'en parlent pas un mot et qu'ils rêvent, comme les autres enfants au demeurant, d'être en mesure d'accéder à l'espace électronique et à ses réseaux sociaux bien connus. Moins les supports légitimes pour se définir sont nombreux, plus les habitants sont sensibles et vulnérables à ceux qui leurs sont proposés par des acteurs institutionnels d'autant plus reconnus et écoutés qu'ils disposent d'un réel pouvoir d'intervention (pour trouver du bois, des cartons, des habits, etc.).

\section{Conclusion}

L'identification de ces trois conditions sociales révèle combien il n'existe pas aujourd'hui dans notre monde contemporain une seule condition sociale qui s'imposerait de fait. L'une des tendances majeures des pays occidentaux est sûrement d'assister à une régression de l'intro-urbanité au profit de l'hétéro-urbanité et de l'extro-urbanité. Par extension et en corollaire, ce sont également des conditions spatiales qui sont en jeu pour comprendre en quoi, parallèlement aux vulnérabilités repérées, il en va d'une remise en cause du commun, ce qui invite à analyser la citoyenneté et ses expressions spatiales inégalitaires (Isin et Wood, 1999).

Partir des trois conditions sociales appréhendées de façon idéal-typique permet de distinguer des territoires théâtres de formes de vulnérabilité plurielles. En cela, nous avons répondu à une triple exigence énoncée au début de cet article $: 1 /$ la vulnérabilité est ici appréhendée à partir d'une pluralité de sources (regards d'autrui, configuration des lieux de vie, représentations politiques, etc.); 2/ elle ne renvoie donc pas uniquement à des dimensions sociales stricto sensu mais également à des dimensions d'ordre bio-psychologique (corporelles par exemple) ou à des conditions externes d'ordre territorial ou morphologique entre autres; et 3/ elle est associée davantage à des situations ou à des territoires qu'à des personnes en tant que telles. Autrement dit, l'analyse des formes de vulnérabilité invite à ne pas occulter la pluralité du monde, d'un monde qui «comprend nos corps, nous-mêmes et notre environnement, autant d'éléments que nous cherchons à relier en un réseau complexe, en soutien à la vie » (Fisher et Tronto, 1990, p. 40).

\section{BIBLIOGRAPHIE}

Agier M. (2015), Anthropologie urbaine, Paris, PUF, 2016.

Bickel J.-F. (2015), « Vulnérabilité, exclusion et politique sociale », in Viriot Durandal J.-P., Raymond E., Moulaert T. et Charpentier M. (dir.), Droits de vieillir et citoyenneté des aînés. Pour une perspective internationale, Québec, Presses de l'université du Québec, p. 297-309. 
Brodiez-Dolino A. (2016), « Le concept de vulnérabilité », La Vie des idées, [en ligne], disponible sur : http://www.laviedesidees.fr/Le-concept-de-vulnerabilite.html.

Butler J. (2010), Ce qui fait une vie. Essai sur la violence, la guerre et le deuil, Paris, Éditions Zones.

Callon M. (1986), « Éléments pour une sociologie de la traduction. La domestication des coquilles Saint-Jacques et des marins-pêcheurs dans la baie de Saint-Brieuc ", L'Année sociologique, $n^{\circ}$ 36, p. 169-208.

Damon J. (2013), Les classes moyennes, PUF.

Daubeuf J.-B. (2018), « L'identité marginalisée : comprendre la "culturalisation" des habitants du bidonville », in Marchal H. et Stébé J.-M. (dir.), Territoires au pluriel, identités au singulier, Paris, L'Harmattan.

Daubeuf J.-B., Marchal H. et Besozzi T. (2017), Idées reçues sur les bidonvilles en France, Le Cavalier Bleu.

Elder G. H. (1994), « Time, human agency and social change : Perspectives on the life course », Social psychology Quaterly, vol. 57, $n^{\circ} 1$, p. 4-15.

Elias N. et Scotson J. L. (1997), Logiques de l'exclusion, Paris, Fayard.

Fisher B. et Tronto J. (1990), « Towards a Feminist Theory of Caring », in Abel E. et Neslon M. (dir.), Circles of Care, New York, SUNY Press, p. 36-54.

Flahault F. (2002), Le sentiment d'exister. Ce soi qui ne va pas de soi, Paris, Éditions Descartes \& Cie.

Foucault M. (1988), « La vie des hommes infâmes », in Dits et écrits, tome III, Paris, Gallimard, p. 237-253.

Giddens A. (1984), The constitution of society, Cambridge, Polity Press.

Goffman E. (1993), Les rites d'interaction, Paris, Éditions de Minuit.

Isin E. et Wood P. K. (1999), Citizenship and Identity, Toronto, Sage.

Lamont M. et Molnär V. (2002), " The study of boundaries in the social sciences ", Annal rewiev of sociology, $\mathrm{n}^{\circ} 29$, p. 167-195.

Lapeyronnie D. avec Courtois L. (2008), Ghetto urbain, Paris, Albin Michel.

Le Blanc G. (2011), Que faire de notre vulnérabilité ?, Paris, Bayard.

Le Breton É. (2008), Domicile-travail. Les salariés à bout de souffle, Paris, Les Carnets de l'Info, 2008.

Le Breton É. (2006), « Homo mobilis », in Bonnet M. et Aubertel P. (dir.), La ville aux limites de la mobilité, Paris, PUF, p. 23-32.

Marchal H. (2017), « Le "vivre-avec" à l'épreuve des divergences urbaines », in Benbassa E. et Attias J.-C. (dir.), Nouvelles relégations territoriales, Paris, CNRS Éditions, p. 53-69.

Marchal H. (2014), « L'épreuve d'inhumanité : l'exemple d'une tour HLM ghettoïsée ", in Boucher M. et Belqasmi M. (dir.), L'État social dans tous ses états, Paris, L'Harmattan, p. 293-304.

Marchal H. et Stébé J.-M. (2017), « Le désenchantement pavillonnaire », SociologieS, en ligne : https://sociologies.revues.org/5957.

Markus H. et Nurius P. (1986), « Possible selves », American psychologist, vol. 21, n 9, p. 954-969.

Martuccelli D. (2014), « La vulnérabilité, un nouveau paradigme ? », in Brodiez-Dolino A, Von Bueltzingsloewen I., Eyraud B., Laval C. et Ravon B. (dir), Vulnérabilités sanitaires et sociales. De l'histoire à la sociologie, Rennes, Presses universitaires de Rennes, p. 27-39. 
Paugam S. (1997), La disqualification sociale, Paris, PUF.

Renahy N. (2005), Les gars du coin, Paris, La Découverte.

Sauvadet T. (2006), Le capital guerrier, Paris, La Découverte.

Sayad A. (1989), Un Nanterre algérien, terre de bidonvilles, Paris, Éditions Autrement.

Soulet M.-H. (2014), « Les raisons d'un succès », in Brodiez-Dolino A., Von Bueltzingsloewen I., Eyraud B., Laval C. et Ravon B. (dir.), Vulnérabilités sanitaires et sociales. De l'histoire à la sociologie, Rennes, Presses universitaires de Rennes, p. 59-64.

Stébé J.-M. et Marchal H. (2016), « Acquérir et rénover une maison en cœur de village. Regards sur un territoire émergent : le pré-urbain », Paris, Ethnologie française, XLVI, n 4 , p. 719-730.

Stébé J.-M. et Marchal H. (2010), Sociologie urbaine, Paris, Armand Colin.

Tarrius A. (2002), La Mondialisation par le bas. Les nouveaux nomades de l'économie souterraine, Paris, Balland.

Tronto J. (2009), Un monde vulnérable. Pour une politique du care, Paris, La Découverte.

Wagner A.-C. (2007), Les classes sociales dans la mondialisation, Paris, La Découverte.

\section{NOTES}

1. C'est à ce niveau que l'analyse de l'agency des individus, entendons leur pouvoir d'agir, prend sens (Giddens, 1984).

2. L'auteur remercie tout particulièrement Jean-Baptiste Daubeuf et Jean-Marc Stébé pour leur précieuse collaboration à deux recherches mobilisées dans cet article. Pour ce qui est des recherches sur le périurbain résidentiel, une quarantaine d'entretiens semi-directifs ont été réalisés entre 2012 et 2016 au sein du périurbain proche et éloigné de la ville de Nancy. Pour plus de précisions sur la méthodologie, nous nous permettons de renvoyer le lecteur à nos travaux (Stébé et Marchal, 2016; Marchal et Stébé, 2017). En ce qui concerne l'étude relative à une tour d'habitat social déshéritée, une observation ethnographique a été menée durant trois mois avec l'aide d'une gardienne d'immeubles. Parallèlement, une dizaine d'entretiens semi-directifs ont eu lieu au domicile des habitants. Là encore, pour plus de précisions sur la méthodologie de la recherche, nous renvoyons le lecteur à nos travaux (Marchal, 2014). Enfin, une observation ethnographique a été réalisée au sein d'un bidonville entre 2013 et 2016, bidonville se trouvant à quelques encablures du centre ville d'une importante métropole régionale de la région Grand Est. Les premiers résultats de cette recherche ont été présentés dans Daubeuf et al. (2017).

3. Aussi ne développerons-nous pas dans le cadre de cet article celles et ceux qui se retrouvent tout ou partie dans la condition de «l'hyper-socialité » (cf. sur ce point Stébé et Marchal, 2010, partie III), dans la mesure où la vulnérabilité y prend, très souvent, d'autres formes que celles liées à leurs territoires de vie légitimés et sécurisés. En effet, les espaces résidentiels privilégiés et huppés de l'hyper-socialité se situent typiquement en centre-ville à proximité des gares ferroviaires et reliés facilement aux aéroports. Il peut aussi s'agir de quartiers gentrifiés plus excentrés ou de poches d'habitat de banlieue (ou même de zones périurbaines) facilement reliées aux centralités urbaines.

4. L'usage des guillemets vise à rappeler qu'en aucun cas il ne s'agit d'êtres humains portant en eux de façon substantielle la condition sociale qui leur est prêtée ici.

5. Les ancrages spatiaux subis et fragiles s'accompagnent ici sans cesse d'une projection de soi dans un ailleurs imaginé, rêvé, via les écrans des smartphones qui procurent l'illusion de pouvoir accéder au monde... 
6. L'usage du «nous » est ici purement conventionnel car il revient plus particulièrement à JeanBaptiste Daubeuf, doctorant au sein du Laboratoire lorrain de sciences sociales (2L2S), d'avoir réalisé ce travail ethnographique dans le cadre d'une thèse de doctorat en sociologie dirigée par l'auteur de ces lignes (cf. pour la méthodologie de la recherche, Daubeuf, 2018).

\section{RÉSUMÉS}

L'article entend mettre en évidence des liens analytiques entre des formes de vulnérabilité et des territoires précis. Il s'appuie sur des recherches empiriques menées en France qui portent aussi bien sur des zones résidentielles excentrées du périurbain, sur une tour déshéritée située dans une cité d'habitat social ou sur un bidonville. Les terrains de recherche retenus sont autant de révélateurs des formes plurielles que prend la vulnérabilité dans la société française actuelle. Sensible à la pluralité des sources et des formes de vulnérabilité, le propos s'attache, chemin faisant, à identifier différents registres de vulnérabilité. Parce que ces différents registres en disent long sur l'existence de fortes disparités non seulement spatiales mais aussi sociales, ils renvoient à des conditions sociales distinctes qui révèlent combien la «société » française contemporaine est le théâtre de profondes divergences socio-spatiales.

The article intends to highlight analytical links between forms of vulnerability and specific territories. It is based on empirical research carried out in France, which includes residential areas, a deprived tower block located in a social housing estate or on a slum. The selected research fields are all revelations of the plural forms that vulnerability takes in the current French society. Sensitive to the plurality of the sources and forms of vulnerability, the aim is to identify the different registers of vulnerability. Because these different registers speak volumes about the existence of strong disparities that are not only spatial but also social, they refer to distinct social conditions that reveal how contemporary French "society" is the scene of profound socio-spatial divergences.

\section{INDEX}

Mots-clés : vulnérabilité, territoires, intro-urbanité, hétéro-urbanité, extro-urbanité

Keywords : vulnerability, territories, intro-urbanity, hetero-urbanity, extro-urbanity

\section{AUTEUR}

\section{HERVÉ MARCHAL}

Professeur de sociologie

Université de Bourgogne-Franche-Comté, LIR3S-UMR 7366 